\title{
A Robust Signal Recognition Method for Communication System under Time-Varying SNR Environment
}

\author{
Jing-Chao LI ${ }^{\dagger}$, Yi-Bing $\mathrm{LI}^{\dagger}$, Nonmembers, Shouhei KIDERA ${ }^{\dagger \dagger \text { a) }}$, and Tetsuo KIRIMOTO ${ }^{\dagger \dagger}$, Members
}

\begin{abstract}
SUMMARY As a consequence of recent developments in communications, the parameters of communication signals, such as the modulation parameter values, are becoming unstable because of time-varying SNR under electromagnetic conditions. In general, it is difficult to classify target signals that have time-varying parameters using traditional signal recognition methods. To overcome this problem, this study proposes a novel recognition method that works well even for such time-dependent communication signals. This method is mainly composed of feature extraction and classification processes. In the feature extraction stage, we adopt Shannon entropy and index entropy to obtain the stable features of modulated signals. In the classification stage, the interval gray relation theory is employed as suitable for signals with time-varying parameter spaces. The advantage of our method is that it can deal with time-varying SNR situations, which cannot be handled by existing methods. The results from numerical simulation show that the proposed feature extraction algorithm, based on entropy characteristics in time-varying SNR situations, offers accurate clustering performance, and the classifier, based on interval gray relation theory, can achieve a recognition rate of up to $82.9 \%$, even when the SNR varies from -10 to $-6 \mathrm{~dB}$.

key words: recognition method for communication signals, entropy characteristic, interval gray relation theory, time-varying SNR environment
\end{abstract}

\section{Introduction}

Appropriate recognition of communication signals [1] is one of the most important and essential technologies, especially for applications such as software radio receivers or general intelligence modems. It plays a significant role in multinetwork communication systems, software radio and radio signal monitoring. Currently, communication systems usually choose different signal modulation types according to the communication environment or desired specifications, with characteristics such as the frequency band, modulation index and system parameters.Depending on these factors, to demodulate the received signal correctly, we need to employ an effective recognition approach to quickly identify the modulation method used by the signal. Thus to ensure the interconnection of different communication systems, the modulation parameters are the most important characteristics in distinguishing the properties of different communication signals. Nowadays, the electromagnetic communica-

Manuscript received February 28, 2013.

Manuscript revised July 19, 2013.

${ }^{\dagger}$ The author are with the Graduate School of Communication and Informatics System, Harbin Engineering University, Harbin, China.

${ }^{\dagger \dagger}$ The authors are with the Graduate School of Informatics and Engineering, The University of Electro-Communications, Chofushi, 182-8585 Japan.

a)E-mail: kidera@ee.uec.ac.jp

DOI: 10.1587/transinf.E96.D.2814 tion environment has become more complex, and the modulation types used in communication signals have diversified. Because the communication environment is not always stable, this study focuses on a method to identify different communication modulation signals in electromagnetic environments with time-varying SNR characteristics.

Feature extraction [2] and classifier design [3] are two important processes in recognizing communication signals. In the process of feature extraction, for the purpose of signal recognition we need to extract the individual characteristics of different modulation signals according to the modulated features. In the literature [4], box counting dimension theory and entropy characteristics are used to extract 2-dimensional characteristic vectors, which offer desirable anti-noise performance. An appropriate threshold process then provides favorable recognition performance to distinguish the communication signals. However, box counting dimension theory is generally more complex than other feature extraction methods because it must divide the signals into different numbers of cells many times, and then calculate the number of cells, to obtain the box counting dimension of the signals. Although the extracted features are stable in the former method [4], it requires much empirical investigation to set an appropriate threshold. Therefore, it is difficult to obtain a high recognition rate when the SNRs are low. The higher order statistics method [5] is currently one of the most effective methods in signal feature extraction. This type of method adopts generalized second order circulation statistics to extract the characteristics with great accuracy. However, even under static SNR conditions, when the SNR is lower than $10 \mathrm{~dB}$, the recognition rate of an OQPSK signal can only exceed $80 \%$, and the recognition rate of BPSK and QPSK signals is under 70\%. Recently developed fractal theory [6] can generally be applied to various image or signal processing issues. However, because the computation is relatively complex, it is not suitable for real-time signal recognition. Entropy features can be used to measure the uncertainty state and the complexity distribution of the signal, and can quantitatively descript the information contained in the signal's characteristics. Because the entropy feature has anti-jamming capabilities for white noise, it is appropriate for communication signal feature extraction under time-varying SNR conditions.

The decision tree classifier [7] is one of the most commonly used classifier design algorithms, but its threshold must be determined precisely. Because of this limitation, it is only suitable for signals with characteristic parameters 
that are sufficiently separated. The neural network classifier [8] has strong adaptive ability, and has been widely used in many fields of classifier design. However, this method requires a priori knowledge of the target signals and also needs a time-consuming training phase. If the overlap of the signals is large, it cannot recognize them properly. By contrast, interval gray relation theory is now widely used in design quality evaluation. This algorithm is an effective method for classifying the characteristics with overlaps. Therefore, even in a time-varying SNR environment, as long as the signals' features do not entirely overlap, it can recognize them better than conventional methods.

To overcome the difficulty mentioned above, this study adopts a feature extraction algorithm based on Shannon entropy and index entropy theory under a time-varying SNR environment. According to the distribution of the extracted features, this study employs interval gray relation theory as a classifier for the different modulation signals, which can realize the purpose of communication signal recognition.

\section{System Model}

There are two important parts to the recognition system of a communication signal: feature extraction and classifier design. After receiving the six communication signals under a $5 \mathrm{~dB}$ time-varying SNR environment, the signals are pre-processed and changed from time domain to frequency domain, and Shannon entropy and index entropy are then used to extract the features of the signals. Finally, the 2dimensional features are submitted to the input of the classifier, and two other types of classifier are used to compare with the interval gray relation theory adopted in this study. The design flow chart of the system is shown in Fig. 1.

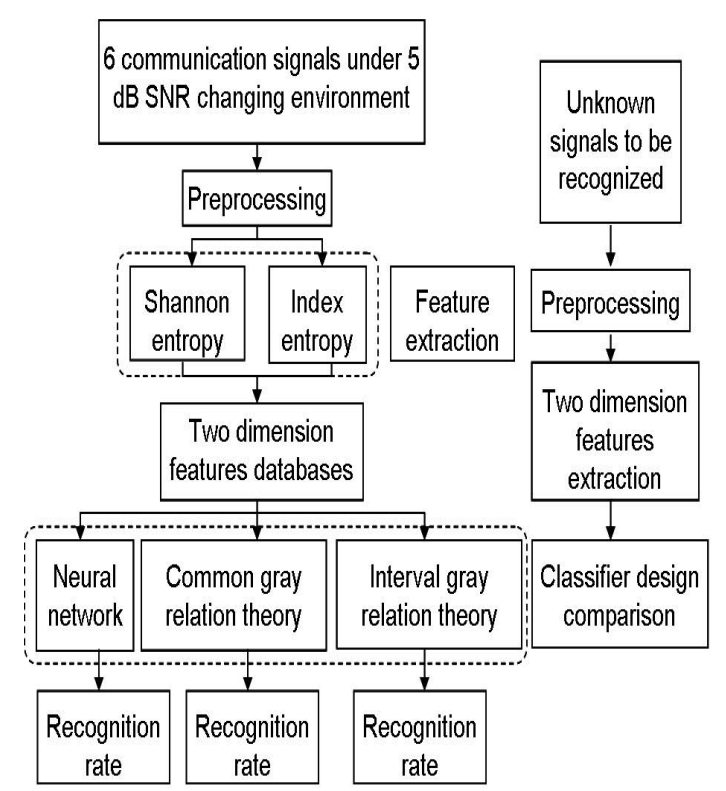

Fig. 1 Flow diagram of the communication signals' recognition system.

\section{Proposed Recognition Method}

Because there is considerable noise in the communication environment, the SNR of the environment is not stable, but changes with time. Therefore, under a time-varying SNR environment, it is difficult to extract the features of the signals precisely. Most of the time, overlaps exist between different features, and it is difficult to design a classifier that can recognize these blurred features. Compared with the stable SNR environment of conventional methods, the timevarying SNR environment is much closer to reality. In addition, the electromagnetic environment has become progressively more complex in the current decade, and there are various types of communication modulation signals whose characteristics are difficult to identify. To address this issue, this study proposes a novel recognition method for communication signals, which is particularly suited to time-varying SNR situations. First, to identify the unknown received signal, we extract two types of characteristics as the Shannon and index entropies, which consist of 2-dimensional feature vectors. Second, we calculate the associated values of the unknown signals with known signals by using the previously prepared database. Finally, we determine the modulation type of the received signals. In the classification stage, this study adopts a classifier based on interval gray relation theory using adaptive weighting [9]. The recognition rate of this method is also compared with different classifiers, which are based on a normal gray relation algorithm and a neural network classifier design algorithm.

\subsection{Feature Extraction Method Based on the Entropy Characteristic}

For a generalized system, entropy can be used to measure the chaos and disorder properties of the state of a system [10]. With regard to the communication signal, it can measure and depict the complexity of different modulation signal characteristics. Generally speaking, when the entropy value is smaller, the degrees of stability, disorder and uncertainty also become smaller. The method for calculating the entropy characteristics of communication signals is as follows:

Step 1) Pre-process the communication signals of different modulation types, supposing the received communication signals to be $s$, and use a 10 times Nyquist sampling rate to sample the signals. The processed signal sequence after discretization is $\{s(n)\}$, where $n=1,2, \cdots, N$ represents the number of sampling points, and $N$ is the length of signal sequence. We then, transform the signal sequence $\{s(n)\}$ from the time domain to the frequency domain using the Fast Fourier Transform (FFT) algorithm:

$$
S(j)=\sum_{n=0}^{N-1} s(n) \exp \left(-j \frac{2 \pi}{N} n j\right) .
$$

After obtaining the frequency spectrum of the signals, we 
calculate the energy value of each point: $E(j)=|S(j)|^{2}$.

Step 2) Because we have obtained the energy value of each point $E(j)$, its probability $p(j)=\frac{E(j)}{E}$ for the energy value of every point $E(j)$ in the whole energy spectrum $E=\sum_{j=1}^{N} E(j)$ can be obtained, which satisfies $0 \leq p(j) \leq 1$, $\sum_{j=1}^{N} p(j)=1$, and $\mathrm{j}=1,2, \cdots, N$, where $N$ is the number of spectrum points;

Step 3)Calculate the Shannon entropy value of the unknown signal as $H_{\mathrm{s}}=-\sum_{j=1}^{N} p(j) \log _{2} p(j)$ and index entropy value as $H_{d}=\sum_{j=1}^{N} p(j) e^{1-p(j)}$, where $\mathrm{j}=1,2, \cdots, N$, and $N$ is the number of spectrum points.

The degree of separation of the Shannon entropy value is generally superior to the index entropy value. This means that if there are two signals with similar probability distribution characteristics, the distance characteristic of the Shannon entropy value is greater than that of the index entropy value. However, there is a problem that when $p(j)=0$, meaning that if there are some spectrum points equal to 0 , the changing information $\Delta I(P(j))=\log (1 / P(j))$ has no meaning and it is then difficult to calculate the changing information of the signals. Adopting index entropy can solve the zero-defined entropy value problem, which will overcome the existing problems of Shannon entropy. The calculation speed of index entropy is quicker than that of Shannon entropy, so it can greatly reduce the computation costs. Because of the advantages of these two entropies from different aspects, this study adopted two entropy value methods to extract the features of the signals, and obtain the combined advantages of the values of two entropy features, which produces a better recognition result.

Using the entropy value characteristics to extract the uncertainty of the signals, the details of the distribution of the signal do not need to be known. The method requires a small amount of calculation and has good anti-noise ability.

\subsection{Classification Using the Interval Gray Relation Theory}

The gray relation algorithm can calculate the degrees of similarity and relation between the parameters of the characteristics of different modulation signals. The modulation signals are then classified according to the degree of relationship in the characteristics. Based on the characteristics of this theory, we adopt the gray relation theory classifier [11] to realize the classification of different communication signals. This theory can be described as follows:

Suppose the system behavior sequence is:

$$
\left[\begin{array}{c}
X_{0}=\left(x_{0}(1), x_{0}(2), \cdots, x_{0}(n)\right) \\
X_{1}=\left(x_{1}(1), x_{1}(2), \cdots, x_{1}(n)\right) \\
\cdots \cdots \\
X_{i}=\left(x_{i}(1), x_{i}(2), \cdots, x_{i}(n)\right) \\
\cdots \cdots \\
X_{m}=\left(x_{m}(1), x_{m}(2), \cdots, x_{m}(n)\right)
\end{array}\right] .
$$

In this study, $X_{0}$ represents the characteristic matrix of the unknown signals, and $X_{i},(i=1,2, \cdots, m)$ represents the characteristic matrix of the known signals in the database. The gray relation coefficient can then be defined as follows:

$$
\gamma\left(x_{0}(k), x_{i}(k)\right)=\frac{\min _{i} \min _{k}\left|x_{0}(k)-x_{i}(k)\right|+\xi \max _{i} \max _{k}\left|x_{0}(k)-x_{i}(k)\right|}{\left|x_{0}(k)-x_{i}(k)\right|+\xi \max _{i} \max _{k}\left|x_{0}(k)-x_{i}(k)\right|} .
$$

Where, $\xi \in(0,1)$ is the distinguishing coefficient, and the value is usually equal to 0.5 . $\gamma\left(x_{0}(k), x_{i}(k)\right)$ is the gray relation coefficient of the $k$ points.

Finally, the degree of the gray relation for $X_{0}$ and $X_{i}$ can be defined as follows:

$$
\gamma\left(X_{0}, X_{i}\right)=\frac{1}{n} \sum_{k=1}^{n} \gamma\left(x_{0}(k), x_{i}(k)\right) .
$$

The procedure for realizing the algorithm follows: Suppose the characteristics samples of the six types of signals are: $H_{\mathrm{mn}}, m=1,2, \cdots, 6, n=s, d$, where $m$ is the number of signals, and $n$ denotes the characteristic type of the signals. In this study each feature is composed of 2dimensional features, namely the Shannon entropy feature $H_{\mathrm{ms}}, m=1,2, \cdots, 6$ as $n=s$, and the index entropy feature $H_{\mathrm{md}}, m=1,2, \cdots, 6$ as $n=d$, which constitute the characteristic matrix:

$$
H_{\mathrm{mn}}=\left[\begin{array}{c}
\mathrm{H}_{1 \mathrm{~s}}, H_{1 d} \\
\mathrm{H}_{2 \mathrm{~s}}, H_{2 d} \\
\vdots \\
\mathrm{H}_{6 \mathrm{~s}}, H_{6 d}
\end{array}\right] .
$$

Set the received unknown signal with 2-dimensional characteristic vectors as $H_{0 \mathrm{n}}=\left(\mathrm{H}_{0 \mathrm{~s}}, H_{0 d}\right)$. This can be used to calculate the gray relation coefficients of the unknown signal characteristics with the known signal characteristics in the database as follows:

$\gamma_{\mathrm{n}}\left(H_{0 n}, H_{m n}\right)=\frac{\min _{m} \min _{n}\left|H_{0 n}-H_{m n}\right|+\xi \max _{m} \max _{n}\left|H_{0 n}-H_{m n}\right|}{\left|H_{0 n}-H_{m n}\right|+\xi \max _{m} \max _{n}\left|H_{0 n}-H_{m n}\right|}$.

Here the value of the distinguishing coefficient $\xi=0.5$. After calculating the gray relation coefficients, the degree of the gray relation can be calculated as follows:

$$
\gamma\left(H_{0 n}, H_{m n}\right)=\frac{1}{2} \sum_{n=d, s} \gamma_{n}\left(H_{0 n}, H_{m n}\right)
$$

Because the simulation condition is under a timevarying SNR environment, even if the extracted characteristic parameters have a certain anti-jamming ability for noise, they will also vary over a certain range, forming a feature interval but not a feature point. According to this characteristic, a classifier based on interval gray relation theory can be designed. 
The procedure for realizing this algorithm is as follows:

Step 1) Each type of signal is repeatedly received with different SNRs, and the changing characteristic interval values of the signals are calculated, namely $H_{\mathrm{mn}}=$ $\left[\left(\mathrm{H}_{\mathrm{ms}}^{\min }, H_{m \mathrm{~s}}^{\max }\right)\left(\mathrm{H}_{\mathrm{md}}^{\min }, H_{m \mathrm{~d}}^{\max }\right)\right]$, and $\mathrm{m}=1,2, \ldots, 6$. Therefore, they can constitute a feature interval matrix.

Step 2) Similarly we repeatedly receive the unknown communication modulation signals to be identified, and calculate the changing characteristic interval values of the signals, namely:

$$
H_{0 \mathrm{n}}=\left[\left(\mathrm{H}_{0 \mathrm{~s}}^{\min }, H_{0 \mathrm{~s}}^{\max }\right) \quad\left(\mathrm{H}_{0 \mathrm{~d}}^{\min }, H_{0 \mathrm{~d}}^{\max }\right)\right] .
$$

Step 3) The characteristic distance between the various communication modulation signals in the database with the unknown signal to be identified, can be calculated as follows:

$$
d_{\mathrm{mn}}=\frac{1}{\sqrt{2}} \sqrt{\left(H_{\mathrm{mn}}^{\min }-H_{0 n}^{\min }\right)^{2}+\left(H_{m n}^{\max }-H_{0 n}^{\max }\right)^{2}} .
$$

We can then obtain the characteristic matrix $D=\left(\mathrm{d}_{m n}\right)_{m \times n}$.

Step 4) The interval gray relation coefficient can then be calculated as:

$$
\varepsilon_{\mathrm{mn}}=\frac{\min _{m} \min _{n}\left\{d_{m n}\right\}+\xi \max _{m} \max _{n}\left\{d_{m n}\right\}}{d_{m n}+\xi \max _{m} \max _{n}\left\{d_{m n}\right\}} .
$$

Thus, we can obtain the interval gray relation coefficient matrix $\zeta=\left(\varepsilon_{m n}\right)_{m \times n}$ of the unknown modulated signal with the known modulation signals.

We then calculate the weighting coefficient of every interval gray relation coefficient. The traditional method is to take the reciprocal of the number of features as the weighting coefficient. This study chose a weighting coefficient based on the entropy algorithm which can improve its adaptive ability.

Step 5)Define $p_{\mathrm{mn}}=\frac{\varepsilon_{\mathrm{mn}}}{\sum_{\mathrm{m}} \varepsilon_{\mathrm{mn}}}$, when $\varepsilon_{m n}=0, p_{m n} \ln p_{m n}=$ 0 . So the method of calculating the entropy value $h_{\mathrm{mn}}$ which is the entropy of the relation coefficient $\varepsilon_{m n}$, is given by:

$$
h_{m n}=-\frac{1}{\ln (n)} \sum_{m} p_{m n} \ln p_{m n} .
$$

where $\ln (n)$ represents the logarithmic function $\log _{e} n$. Thus, the weighting value definition of the coefficient characteristics $a_{m n}$ is:

$$
a_{\mathrm{mn}}=\frac{1-h_{m n}}{\sum_{n}\left(1-h_{\mathrm{mn}}\right)},
$$

and $a_{m n} \geq 0, \sum_{\mathrm{n}} a_{m n}=1$.

Therefore, the degree of the gray relation:

$$
r_{\mathrm{m}}=\sum_{n} \varepsilon_{m n} \cdot a_{m n},
$$

where $r_{\mathrm{m}}$ indicates the degree of the gray relation of the $m$ signal in the database with the signal to be identified. The bigger $r_{\mathrm{m}}$ is, the greater the probability of the unknown signal belonging to the $m$ signal. Conversely, the smaller $r_{\mathrm{m}}$, the smaller the probability of the unknown signal belonging to the $m$ signal.

\section{Results from Numerical Simulations}

Compared with the conventional methods of signal recognition in an environment of a stable SNR, this study chose a $5 \mathrm{~dB}$ SNR time-varying white noise environment $(0 \mathrm{~dB}-4$ $\mathrm{dB}, 3 \mathrm{~dB}-7 \mathrm{~dB}, 5 \mathrm{~dB}-9 \mathrm{~dB}, 9 \mathrm{~dB}-13 \mathrm{~dB}$ were selected in this study), and six different communication modulation signals were then selected: Amplitude Modulation (AM), Frequency Modulation (FM), Phase Modulation (PM), Amplitude Shift Keying (ASK), Frequency Shift Keying (FSK), Phase Shift Keying (PSK). The modulation parameters of the six signals were selected as follows:

The carrier frequency $f_{z}=2.7 \times 10^{8} \mathrm{~Hz}$, sampling frequency $f_{s}=4.32 \times 10^{9} \mathrm{~Hz}$, and baseband signal frequency $f_{m}=1.0 \times 10^{5} \mathrm{~Hz}$. For the analog modulation signals the $\mathrm{AM}, \mathrm{FM}$ and PM signals were the same. For the AM signal, the amplitude was $A=1$, and the amplitude-modulation index was $a=0.8$. For the FM signal, the frequencymodulation index was $k_{f}=6$ and for the PM signal, the phase-modulation index was $k_{p}=5$. The carrier frequency was $f_{z}=2.7 \times 10^{8} \mathrm{~Hz}$ and the symbol rate $R_{b}=1.0 \times 10^{5} \mathrm{bps}$ for the digital modulation signals ASK, FSK, PSK were the same. The ASK was 0,1 keying signal. The two frequencies of the FSK signal were $f_{1}=f_{z}-\Delta f$ and $f_{2}=f_{z}+\Delta f$, $\Delta f=1.0 \times 10^{5} \mathrm{~Hz}$. And $0, \pi$ binary phase-modulation was used for the PSK signal.

As previously described, we extracted two types of entropy value characteristics for the six signals when the SNR was varying with time, constituting a 2-dimensional character space. Simulation results are shown in Fig. 2, where the abscissa $H s$ represents the Shannon entropy, the ordinate $H d$ represents the index entropy, and the sub-graphs (a), (b), (c), (d) are under different SNR environments. It can be seen in Fig. 2 that for a lower SNR, different communication modulation signals had greater overlapped space. Under the $0 \mathrm{~dB}-4 \mathrm{~dB}$ environment, the interval of the features for the two signals FSK and PSK have a significantly overlapped area, so it is difficult for an ordinary classifier to classify correctly. However, for the interval gray relation classifier, because it uses the interval of the characteristics of the signals for identification, and as long as the characteristics of the intervals of the two types of signals are not completely overlapped, the unknown signal can be recognized and classified by this classifier with a recognition rate of $99.6 \%$.

Under a time-varying SNR environment, the communication modulated signals will be repeatedly received 20 times, and the Shannon entropy and index entropy characteristics of the received signals will also be extracted 20 times, so the entropy features interval under this time-varying SNR can be acquired. We compared this method with a tradi- 
Table 1 Recognition rate of different classifier algorithm under variance SNR environments.

\begin{tabular}{|c|c|c|c|c|c|c|}
\hline SNR(dB) & $9 \sim 13$ & $5 \sim 9$ & $3 \sim 7$ & $0 \sim 4$ & $-5 \sim-1$ & $-10 \sim-6$ \\
\hline Interval gray relation theory's recognition rate (\%) & 100 & 100 & 100 & 99.6 & 97.1 & 82.9 \\
\hline Normal gray relation theory's recognition rate (\%) & 99.2 & 98 & 95.8 & 82.7 & 64 & 43 \\
\hline Neural network's recognition rate (\%) & 67.3 & 55 & 51.9 & 47.3 & 40.6 & 32.3 \\
\hline
\end{tabular}
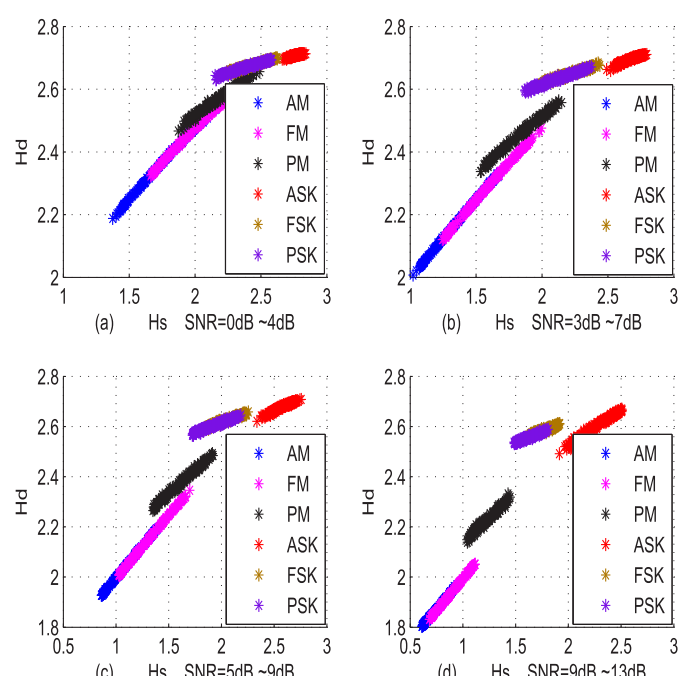

Fig. 2 Entropy characteristics of six communication signals under timevarying SNR environment.

(a) SNR is changed from $0 \mathrm{~dB}$ to $4 \mathrm{~dB}$, (b) SNR is changed from $3 \mathrm{~dB}$ to $7 \mathrm{~dB}$, (c) SNR is changed from $5 \mathrm{~dB}$ to $9 \mathrm{~dB}$, (d) SNR is changed from $9 \mathrm{~dB}$ to $13 \mathrm{~dB}$. Hs represents the Shannon entropy of different signals, $H d$ represents the index entropy of different signals.)

tional gray relation classifier [11] and the neural network classifier algorithms [12], and then calculated the recognition results for different classifiers. The simulation results are shown in Table 1, which indicates that because the neural network classifier needs to be trained using every feature point of the different signals, the received signal was classified according to the distribution features of the feature points. For the overlapping features of the signals, the neural network classifier does not have any recognition ability, so this is the worst result. For the ordinary gray relation classifier, when the overlap area is small, it is very effective. However, when the overlap area is greater, the recognition rate falls rapidly, and it is therefore not useful for recognition in low SNR conditions.

The interval gray relation classification algorithm was adopted in this study with a characteristic interval to be identified because of its good recognition capability. Even under a SNR from $-10 \mathrm{~dB}$ to $-6 \mathrm{~dB}$, it could still achieve a recognition rate of $82.9 \%$. Because the interval relation classifier adopts the method of repeatedly receiving the signals, it takes more time to identify them. Comparison of the simulation time of the interval gray relation algorithm with normal gray relation theory is shown in Table 2 . The simulation results were calculated using a household computer with an Intel $2.4 \mathrm{GHz}$ dual processor.

From the simulation results in Table 2 it can be seen that the simulation time for the interval gray relation clas-
Table 2 Simulation time of interval gray relation algorithm and normal gray relation theory.

\begin{tabular}{|c|c|c|}
\hline Algorithm & Normal gray relation theory & Interval gray relation theory \\
\hline Time (s) & 1.50 & 24.58 \\
\hline
\end{tabular}

sifier is 16.4 times longer than for the normal relation algorithm. It requires a longer simulation time because in this study it receives the signal 20 times repeatedly.In a practical engineering application, if there is no strict requirement on time, it is acceptable to use the interval gray relation classifier algorithm to obtain better identification results. If there is a requirement to limit time, and the SNR is not very low, we can use the ordinary gray relation algorithm, which has a higher calculation speed.

\section{Conclusions}

A new communication signal recognition method was proposed in this study, and the two key links in the recognition system, i.e. feature extraction and classifier design, were simulated and analyzed. Simulation results demonstrated that the feature extraction algorithm based on Shannon entropy and index entropy has a better anti-noise performance, and it maintains a better clustering performance even in low SNR environments. According to the distribution characteristics of the entropy under time-varying SNR, the classifier algorithm based on interval gray relation theory can make full use of the interval characteristics of the signals, and have an improved classification effect compared with that obtained by conventional methods.

\section{References}

[1] A.K. Nandi and E.E. Azzouz, "Algorithms for automatic modulation recognition of communication signals," IEEE Trans. Commun., vol.46, no.4, pp.431-436, 1998.

[2] B. Ghoraani and S. Krishnan, "Time frequency matrix feature extraction and classification of environmental audio signals," IEEE Trans. Audio Speech Language Process., vol.19, no.7, pp.21972209, 2011.

[3] Y. Lin and J.-C. Li, "Radar signal recognition algorithms based on neural network and gray relation theory," Cross Strait QuadRegional Radio Science and Wireless Technology Conference (CSQRWC), pp.1482-1485, 2011.

[4] Y.-B. Li, J.-C. Li and Y. Lin, "The identification of communication signals based on fractal box dimension and index entropy," J. Convergence Information Technology, vol.6, no.11, pp.201-208, 2011.

[5] C.-H. Zhao, W.-C. Yang and S. Ma, "Research on communication signal modulation recognition based on the generalized secondorder cyclic statistics," J. Communications, vol.32, no.1, pp.144150, 2011. (in Chinese)

[6] S. Gunasekaran, and K. Revathy, "Fractal dimension analysis of audio signals for Indian musical instrument recognition," International Conference on Digital Object Identifier., pp.257-261, 2008. 
[7] X. Duan and H. Xue, "Multi-decision-tree classifier in master data management system," International Conference on Business Management and Electronic Information (BMEI), pp.756-759, 2011.

[8] J. Gaeddert and J.H. Reed, "A new approach to signal classification using spectral correlation and neural networks," 2005 First IEEE International Symposium on New Frontiers in Dynamic Spectrum Access Networks., pp.144-150, 2005.

[9] Y.-B. Li, J.-C. Li, and J. Kang, "Classifier design algorithms aimed at overlapping characteristics," Information Technology Journal., vol.11, no.8, pp.1091-1096, 2012.

[10] J. Li, Y. Li, and Y. Lin, "The application of entropy analysis in radiation source feature extraction,” J. Projectiles, Rockets, Missiles and Guidance, vol.31, no.5, pp.155-160, 2011. (in Chinese)

[11] Y. Lin, X.-C. Si and R.-L. Zhou, "Application of improved gray correlation algorithm on radiation source recognition," Journal on Communications., vol.31, no.8A, pp.166-171, 2010 (in Chinese)

[12] H.C. Dubey, Nandita, and A.K. Tiwari, "Blind modulation classification based on MLP and PNN," 2012 Students Conference on Engineering and Systems (SCES), pp.1-6, 2012



Jing-Chao Li received the B.S. in Electrical Information Engineering from Harbin Engineering University(HEU) in 2010. During 2010-2011, she stayed in HEU to read M.S., During 2011-2012, she stayed in HEU to read P.D. During 2012-2013, she was in University of Electro-Communication (UEC) as exchanged student. Now she is studying communication signals recognition.

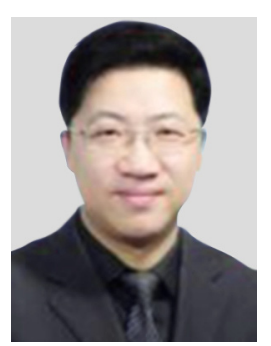

Yi-Bing $\mathbf{L i}$ received the B.S. and M.S. and Ph.D degrees in Harbin Marine engineering college, Harbin engineering university in 1989, 1997 and 2003, respectively. He has been a teacher in Harbin Engineering University of China since 1989, and became as professor in 2004. During 2007-2008, he stayed in the University of Hong Kong Electronic Engineering lab as a visiting scholar. Now he is a IEEE member, a senior member of China communication and a China computer association member.

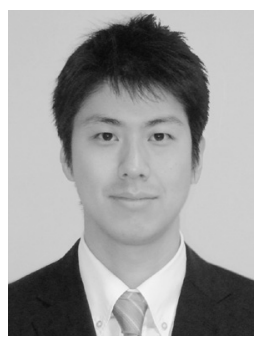

Shouhei Kidera received his B.E. degree in Electrical and Electronic Engineering from Kyoto University in 2003 and M.I. and Ph.D. degrees in Informatics from Kyoto University in 2005 and 2007, respectively. He is an assistant professor in Graduate School of Informatics and Engineering, University of ElectroCommunications, Japan. His current research interest is in advanced signal processing for the near field radar, UWB radar. He is a member of the Institute of Electrical and Electronics Engineering (IEEE) and the Institute of Electrical Engineering of Japan (IEEJ).

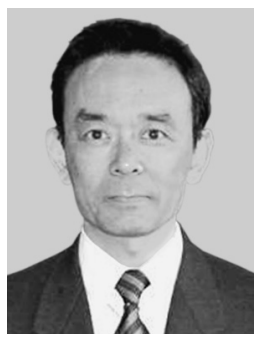

Tetsuo Kirimoto received the B.S. and M.S. and Ph.D degrees in Communication Engineering from Osaka University in 1976, 1978 and 1995, respectively. During 1978-2003 he stayed in Mitsubishi Electric Corp. to study radar signal processing. From 1982 to 1983 , he stayed as a visiting scientist at the Remote Sensing Laboratory of the University of Kansas. From 2003 to 2007 , he joined the University of Kitakyushu as a Professor. Since 2007, he has been with the University of Electro-Communications, where he is a Professor at the Graduate School of Informatics and Engineering. His current study interests include digital signal processing and its application to various sensor systems. Prof. Kirimoto is a senior member of IEEE and a member of SICE (The Society of Instrument and Control Engineers) of Japan. 